\title{
The role of the family in the psychiatric treatment dropout
}

\section{DOI: http://doi.org/10.26758/7.1.5}

Eugjen Sotiri, Fatime Elezi, Ardian Braho, Lindita Alushi

Psychiatric Emergency, University Hospital Center Tirana “Mother Teresa”, Albania

Address correspondence to: Eugjen Sotiri, Shërbimi i Psikiatrisë, QSUT “Nënë Tereza”, Rr. Dibrës 372 Tirana, Albania. Ph.: +355692024996; e-mail: esotiri@ gmail.com

\begin{abstract}
Objectives. The aims of our study were to classify the reasons for psychiatric treatment dropout. Materials and methods. 300 patients diagnosed with schizophrenia and bipolar I disorders who had discontinued psychiatric treatment were questioned about the reasons for discontinuation during 2014. Reasons included the report of the patient and conclusions drawn by the psychiatrists based on the whole context of the patient's life and family reports. The study sample included only patients with at least two hospital admissions, in order to make sure they had some experience with psychiatric disorders, psychiatric treatment and were under specialized treatment, thus a confirmed diagnosis.

Results. The first reason of dropout is reported as follows: $36.6 \%$ of dropout results due to lack of insight; $31.7 \%$ due to side effects; $8.4 \%$ for economic reasons; $23.3 \%$ after significant improvement of the symptoms. $79 \%$ of subjects revealed that the second reason is related to family care and support. This derives from two main causes: $42 \%$ because of continuous poor family support, related to the socioeconomic status, and $37 \%$ due to decrease in family support, mainly as a result of parents' loss or aging, or emigration of siblings.

Conclusions. Dropout of psychiatric treatment brings a lot of challenges for the mental health system. Many of the factors are changeable. Dropout factors vary from disorder-related to socialrelated issues. Primary reasons are related to the disorders and medication; family-related causes seem to be very important factors that influence dropout from psychiatric treatment. Professional workers and family care should be better oriented for an overall better mental health care.
\end{abstract}

\section{Keywords: treatment dropout; family support; hospital admission; socioeconomic status.}

\section{Introduction}

Medical treatment adherence, as an important element for a successful therapeutical management, especially for chronic conditions is studied and discussed for all medical specialties. It has also economic implications directly related to poor health and decreased performance (Osterberg and Blaschke, 2005). In addition, we should be careful to avoid errors of adherence. Errors in drug adherence may be categorized into four groups: errors of omission, errors of purpose (taking medicine for the wrong reasons), errors of dosage, and mistakes in timing or sequence. There is another group of patients who takes additional medications not prescribed by the physician, which may be potentially dangerous (Blackwell 1976). Psychiatric patients are considered to be the highest non-adherent patients because of stigma and cognitive changes. Psychiatrists may also provide more expertise in other fields of medicine about this issue. There are two direct reasons why psychiatrists 
need to concern themselves with the problems of adherence. The first is the implication it has for personal practice; another is to provide expert consultation to other health professionals on the management of adherence in general medical practice. Two of the important contributions of psychiatry to the rest of medicine may be a better understanding of the extent to which treatment is specific and of the degree to which treatment can be implemented (Blackwell, 1976). These conclusions, made about forty years ago, are still relevant. Further studies have implied much more implications. This includes also socioeconomic reasons. One of the non-adherence models is drug treatment dropout (Blackwell, 1976). There are many risk factors for dropout. The behavioral model of access to health care offers a framework for organizing findings on predictors of dropout. Dropout risk can be viewed as a joint function of predisposing demographic factors (gender and age), social factors (marital status and education), enabling factors (health insurance, income, and number of providers), and needs (psychiatric disorder, co-morbidities, and past treatment) (Olfson et al., 2009).

With reference to the specifics of Albania, we believe that many of the latest changes and reforms have affected mental health system and the mentality about mental health may not be quite coherent with these changes. There has been a debate between those who favor providing mental health treatment and care in hospitals, and those who prefer providing it in community settings, primarily or even exclusively. A third alternative is to utilize both community services and hospital care. In this balanced care model, the focus is on providing services in normal community settings close to the population served, while hospital stays are as brief as possible, promptly arranged and used only when necessary. This balanced interpretation of community-based services goes beyond the rhetoric about whether hospital care or community care is better and instead encourages consideration of what blend of approaches is best suited to a particular area at a particular time. Primary care staff will probably need to provide most if not all of the mental health services in primary health care settings, with specialist backup to provide training, consultation, inpatient assessment and treatment that cannot be provided in primary care. Some low-resource countries may, in fact, be in a pre-asylum stage, in which the apparent community care, in fact, represents widespread neglect of the mentally ill (Thornicroft and Tansella, 2003). The major recent change in mental health care system in Albania is the development of community mental health care. This strategy, which has been introduced since the early 2000s, started to be implemented later. Considering the difficulties of a country in development, it has not been fully put into effect. The first rapid developments have been followed by a plateau phase in the implementation. Logistic, financial and human resources factors contribute to this deceleration. This situation has influenced the treatment of mentally ill patients, as the follow-up of a patient in the community is important for the medical treatment and social inclusion with direct consequences on the overall functioning of mental health patients. However, this process will continue and mental health services will provide better care for the patients. Key issues of the strategy to provide better care include further development of psychosocial services and performance indicators for mental health services (Ministry of Health Albania, 2013). On the other hand, if community services are not quite developed or lack adequate human and logistic resources, the role of the family in supporting mental health patients with or without function decreases. Primary care system also offers only rudimentary mental health care and is not contributing quite enough for the treatment.

In this context, it is very important to explain the mentality of Albanian society related to family connections. Traditionally, the Albanian family has been the primary source of support for its members. This support includes patient support and in the case of a psychiatric patient, it has a greater role because of the lack of insight of a psychiatric patient compared to a patient with a medical condition. This approach has increased the total burden of care for the families (Leggatt, 
2002). Traditionally, large psychiatric hospitals have been shelters for a wide range of mentally ill patients (Leggatt, 2002). This approach has ensured the drug treatment of these patients, but it has not offered multidisciplinary treatment, which may also help psychiatric patients to preserve skills and to develop new ones. Community care intends toward a multidisciplinary approach for the mentally ill persons and also for the inclusion of these patients in the society, but Albania suffers from insufficiency of human resources and population coverage (WHO Aims Report, 2006), thus family burden is increased and family care has to take a greater role in the management of patients. The rate of hospitalization decreased initially, but it started to increase again, due to increased family burden. This results in loss of energy and leads to poor adherence and dropout of psychiatric treatment, which seems to be an important factor for the increase of hospitalization rate and the overall quality of life for the mentally ill patients (Tehrani et al., 1996; Masaki et al., 1997; Furegato, Castro and Santos, 2011). In the Albanian context, the family has a crucial role in the management of patients. There are also strong relations with the role of family care, psychiatric treatment dropout, psychiatric hospitalizations and quality of life of the patients (Furegato, Castro and Santos 2011; Baekeland and Lundwall, 1975; Chen, 1991).

\section{Materials and methods}

This is an observational study with descriptive components. The study included 300 patients who came for consultation in the hospital's psychiatric service in Tirana, Albania, during 2014. Subjects were patients suffering from schizophrenia and bipolar I disorder diagnosed according to DSM-IV-TR criteria, and having discontinued the treatment. At least two hospital admissions were among the selection standards to meet, in order to make sure that the patients have some experience with psychiatric disorders, psychiatric treatment and were under specialized treatment, thus a confirmed diagnosis. Another requirement was that patients should respond directly about treatment discontinuation, so the physician could have sufficient information about the life and treatment context in order to draw proper conclusions. The study also included patients who were consulted directly because of a relapse of symptoms and whose treatment was discontinued against medical advice. Patients that have not answered clearly about the reason for discontinuation and without adequate information about life context have been excluded.

To the patients' group have been posed direct questions about the cause of treatment discontinuation, and the answer has been given directly by each patient. This answer has been considered as the first reason for treatment dropout. The responses were grouped into the following categories: "I do not have money to buy medicines" - economic reasons, "I am not ill" - lack of insight, "I am very well now, why to take medicines" - improvement of symptoms (in this case we have been careful to clarify improvement of symptoms with lack of insight), "since I have taken this medicine, I have some new problems" - side effects (which have been also evaluated as side effects by the examining psychiatrist).

A group of questions about family and life context has been directed to the patient, family members, and/or accompanying person. Also, conclusions from referral documents have been carefully examined in order to have a clear picture of family involvement in the care of patients, for example: Do you take your medication yourself or someone other gives it to you? Answer: "My sister comes to my home every day and gives it to me", or: "Why after many years of maintained treatment the patient has discontinued it in this proper moment?" Answer: "Till now the mother of the patient has given the medicines rigorously, but she passed away three months ago". This group of questions also obtained data about the socioeconomic status of the patient and the family. Considering the fact that there is not an adopted scale of measuring the socioeconomic status in the 
country, even with its limitations, we have "classified" all family socioeconomic situations in three groups related to monthly family income: low level, $<200$ euro (146 subjects), medium level, 200700 euro, (96 subjects) and high level, > 700 euro monthly family income (58 subjects). Another classification is related to rural and urban inhabitants.

\section{Results}

The results were classified into two major categories of reasons. The first is related to direct reasons of dropout medication without taking into consideration the context of the patient's life. Beyond the direct reasons for discontinuation, evaluation of the context of the patient's life results in the direct implication of the family as an important factor for the dropout of psychiatric treatment. The vast majority of cases revealed that the second reason for the first three groups (79\% of all respondents) is related to family care and support (Table 1).

Table 1. Results of dropout reasons

\begin{tabular}{|l|c|c|c|c|c|c|c|c|c|c|}
\hline \multicolumn{1}{|c|}{$\begin{array}{c}\text { Reasons of } \\
\text { dropout }\end{array}$} & \multicolumn{2}{|c|}{ Second reason } & \multicolumn{2}{c|}{$\begin{array}{c}\text { Lack of family } \\
\text { support }\end{array}$} & $\begin{array}{c}\text { Decrease of } \\
\text { family } \\
\text { support }\end{array}$ & \multicolumn{2}{c|}{$\begin{array}{c}\text { Medical } \\
\text { reasons }\end{array}$} & \multicolumn{2}{c|}{ Other } \\
\hline First reason & \multicolumn{2}{|c|}{ Total } & $42.0 \%$ & 126 & $37.0 \%$ & 111 & $11.0 \%$ & 33 & $10.0 \%$ & 30 \\
\hline Lack of insight & $36.6 \%$ & 110 & $17.0 \%$ & 51 & $13.0 \%$ & 39 & $1.7 \%$ & 5 & $5.0 \%$ & 15 \\
\hline Side effects & $31.7 \%$ & 95 & $14.0 \%$ & 42 & $11.6 \%$ & 35 & $3.7 \%$ & 11 & $2.4 \%$ & 7 \\
\hline $\begin{array}{l}\text { Improvement of } \\
\text { symptoms }\end{array}$ & $23.3 \%$ & 70 & $8.7 \%$ & 26 & $9.7 \%$ & 29 & $5.0 \%$ & 15 & $0.0 \%$ & 0 \\
\hline $\begin{array}{l}\text { Economic } \\
\text { reasons }\end{array}$ & $8.4 \%$ & 25 & $2.3 \%$ & 7 & $2.7 \%$ & 8 & $0.6 \%$ & 2 & $2.6 \%$ & 8 \\
\hline
\end{tabular}

Poor or discontinuous family support is reflected as the involvement of family members in the distress that a particular patient produces to them. Usually, as reported, family members get involved only if the patient affects their lives directly (e.g.: aggressive behavior inside or outside the home). In most of the cases, the family members are satisfied if the patients do not pose risk to them. The lower socioeconomic level of family members resulted in higher dropout rates and nearly half of all subjects (48.6\%) are in this group, while medium socioeconomic group includes $32 \%$ of all subjects. On the other hand, the higher socioeconomic level group $(19.3 \%)$ has the lowest number of subjects, consequently reflecting a better level of support. Also, rural area inhabitants show a poor level of support (59\%). In this last case, stigma, lifestyle and education level are also some of the components that hinder social inclusion of patients. All these factors imply that socioeconomic status of the patient and his family has an important effect in treatment dropout (Table 2). 
Table 2. Socioeconomic relations

\begin{tabular}{|l|c|c|c|c|c|c|c|c|}
\hline $\begin{array}{c}\text { Socioeconomic } \\
\text { relations }\end{array}$ & \multicolumn{2}{|c|}{ Income } & \multicolumn{2}{c|}{ Low } & \multicolumn{2}{c|}{ Medium } & \multicolumn{2}{c|}{ High } \\
\hline & \multicolumn{2}{|c|}{ Total } & $48.6 \%$ & 146 & $32.0 \%$ & 96 & $19.3 \%$ & 58 \\
\hline Rural & $59 \%$ & 177 & $34.0 \%$ & 102 & $18.0 \%$ & 54 & $7.0 \%$ & 21 \\
\hline Urban & $41 \%$ & 123 & $14.6 \%$ & 44 & $14.0 \%$ & 42 & $12.0 \%$ & 37 \\
\hline
\end{tabular}

Another observation is that many of the patients with good support in their personal history, as reported by caregivers or referral documents (37\%), have a decrease in family support as a result of a new family situation. For a considerable percentage of patients (30\%), the challenge is represented by a parent's loss, while for a smaller ratio (13.5\%) by aging of the parents, who cannot longer provide the same level of care. The rest of this group $(56.5 \%)$ has had support from their siblings, whose emigration has resulted in decreased support for these patients.

\section{Discussion}

The above-mentioned observational data with simple descriptive evaluation make us aware of the limitations of this study. The resulted data show that factors influencing psychiatric treatment dropout range from purely psychiatric to purely social, as well as overlaps between them (Osterberg and Blaschke, 2005). On the other hand, literature and studies in other countries sometimes provide different conclusions and also contradictory conclusions about dropout rates and predictors (Ribeiro et al., 2012), which may also indicate that in different cultures and conditions the evaluation of a situation should probably be started from different assumptions (Reneses et al., 2009). Data for this evaluation have been gathered only in one hospital. Other studies point out hat even in welldesigned studies national surveys should be performed for more comprehensive data and results (Olfson et al., 2009). There are studies that report a higher number of dropouts in lower socioeconomic level patients (Bruwer et al., 2011), and also studies that reveal a higher percentage of dropouts related to the lower socioeconomic status of their families (Pelkonen et al., 2000). Similar findings related to the impact of the socioeconomic level were obtained within the current study. Considering the outcomes of treatment dropout especially in the group of patients suffering from psychotic disorders, it is very important to raise the awareness of psychiatrists and to work and educate family members of patients to detect early signs of treatment dropout (Hui et al., 2000). Considering the fact that lack of insight is the most frequent factor in our observation compared to international data, this seems to be somewhat higher than other countries. This situation may be explained by the heavy workload of the psychiatrists in Albania and rudimentary multidisciplinary approach.

However, this is the first attempt to evaluate dropout factors and the relation with the family support in Albania. As literature indicates, there are many overlaps between various locations about the same issues related to dropout of treatment, also there are some local factors especially related to the family connections and to the specific situation in Albania. As previously mentioned, these specificities concern the rudimentary support within primary care facilities for mental health services and the shortage of community mental health care. In this context, this evaluation assessment serves as a cornerstone for further well-designed studies with stronger conclusions. 


\section{Conclusions}

Dropout of psychiatric treatment brings a lot of challenges for the mental health system. Factors influencing the phenomena derive from many parts of the care system for the mental illnesses. Many of the classified reasons are highly probable to be changed. Lack of insight, especially for the studied disorders, is directly related to the work of the multidisciplinary staff within the mental health system. In addition focusing on side effects and choosing drugs with the fewest side effects possible or lowering side effects by using the lowest appropriate dose prescribed by the physicians will help patients to continue effective treatment. Family support, even if not in the first sight of the problem, is present in one form or another in the majority of cases. In this context, demographic changes should be foreseen as accurately as possible in order to take the necessary measures to ease the social and economic burden. Albanian family has still a great solidarity, but there are also other reasons that go beyond. As reported, aging of the parents of the mentally ill patients has started to produce effects in the care and quality of life for the patients, so measures to replace the support of parents should be taken by the social services. Also, emigration has given its consequences with decreasing the level of care. The socioeconomic status has its implications and this point should be taken into consideration when evaluating the total cost of mental health disability. Finally, this attempt should be followed by other studies with the support of government organizations, including costs evaluation for medicines and social support.

\section{Bibliography}

1. Baekeland, F., Lundwall, L., 1975. Dropping out of treatment: a critical review, Psychological Bulletin, 82 (5), pp. 738-783.

2. Blackwell, B., 1976. Treatment Adherence. British Journal of Psychiatry, 129 (6), pp.513-531.

3. Bruwer, B, Sorsdahl, K, Harrison, J., Stein, D.J., Williams, D., Seedat, S., 2011. Barriers to mental health care and predictors of treatment dropout in the South African Stress and Health Study. Psychiatric Services, 62 (7), pp. 774-781.

4. Chen, A., 1991. Noncompliance in community psychiatry: a review of clinical interventions. Hospital \& Community Psychiatry, 42 (3), pp. 282-287.

5. Furegato, A.R.F., Castro, S.A., Santos, J.L.F., 2011. Psychiatric readmissions and their association with sociodemographic and clinical profiles. Journal of Epidemiology \& Community Health, A362 doi: 10.1136/jech.2011.142976m.39.

6. Hui, C.L., Chen, E.Y., Kan, C.S., Yip, K.C., Law, C.W., Chiu, C.P., 2006. Detection of nonadherent behavior in early psychosis. Australian \& New Zealand Journal of Psychiatry, 40 (5), pp. 446-451

7. Leggatt, M., 2002. Families and mental health workers. The need for partnership. World Psychiatry, 1 (1), pp. 52-55.

8. Masaki, N., Fujita, T., Kai, S., Zaitsu, Y., Hira, Y., Kondoh K., Yamashita, K., Hatada, K., 1997. Readmission among discharged psychiatric patients and it's correlates. Japanese Journal of Public Health - Nihon Koshu Eisei Zasshi, 44 (5), pp. 372-383.

9. Olfson, M., Mojtabai, R., Sampson, N.A., Hwang, I., Druss, B., Wang, P.S., Wells, K.B., Pincus, H.A., Kessler, R.C., 2009. Dropout from Outpatient Mental Health Care in the United States. Psychiatric Services, 60 (7), pp. 898-907.

10. Osterberg, L., Blaschke T., 2005. Adherence to medication. New England Journal of Medicine, 353 (5), pp. 487-497. 
11. Pelkonen, M., Marttunen, M., Laippala, P., Lönnqvist, J., 2000. Factors associated with early dropout from adolescent psychiatric outpatient treatment. Journal of the American Academy of Child \& Adolescent Psychiatry, 39 (3), pp. 329-336.

12. Reneses B., Muñoz, E., López-Ibor, J.J., 2009. Factors predicting drop-out in community mental health centres. World Psychiatry, 8 (3), pp. 173-177.

13. Ribeiro, M.S., Xavier Junior J.C.C., Mascarenhas T.R., Silva M.P., Melo Vieira E.M., Ribeiro L.C., 2012. Treatment dropout at a secondary mental health service. Trends in Psychiatry and Psychotherapy, 34 (4), pp. 207-214.

14. Tehrani, E., Krussel, J., Borg, L., Munk-Jørgensen, P., 1996. Dropping out of psychiatric treatment: a prospective study of a first-admission cohort. Acta Psychiatrica Scandinavica, 94 (4), pp. 266-271.

15. Thornicroft, G., Tansella, M., 2003. What are the arguments for community-based mental health care? Copenhagen, WHO Regional Office for Europe, Health Evidence Network report, Available from: $\leq$ http://www.euro.who.int/document/E82976.pdf $\geq$, [Accessed 29 November 2015]

16. ***Ministry of Health Albania, 2013. Action plan for development of mental health services in Albania 2013-2022. Available from: 〈http://www.shendetesia.gov.al/files/userfiles/Baza_Ligjore/Dokumenta_strategjike/pv1.pdf〉 [Accessed 8 November 2015]

17. ***WHO-AIMS Report on Mental Health System in Albania. WHO and Ministry of Health, Tirana, Albania, 2006. Available from: 〈http://www.who.int/mental_health/albania_who_aims_report1a.pdf $>$ [Accessed 10 November 2015] 